http://dx.doi.org/10.18232/alhe.983

Artículos

\title{
Dificultades y contradicciones en la construcción del socialismo en Cuba al inicio del periodo revolucionario
}

\section{Difficulties and contradictions in the construction of socialism in Cuba at the beginning of the revolutionary period}

\author{
Bruno Sovilla $1, *$ iD http://orcid.org/0000-0002-1148-6670 \\ ${ }^{1}$ Universidad Autónoma de Chiapas, San Cristóbal de Las Casas, México. \\ *Correspondencia: brunosov@hotmail.com
}

Resumen. En este trabajo pretendemos demostrar que las dificultades económicas al inicio del periodo revolucionario radicaron inicialmente en una estrategia de desarrollo mal diseñada, y sucesivamente en la implementación de un sistema de dirección de la economía que prescindía no solo del mercado, sino también de los principios del cálculo económico, a la base de la planificación centralizada. Después de analizar la información estadística oficial y el debate que tuvo lugar en Cuba a mitad de los años 60 sobre el sistema de dirección más adecuado para una economía en vías de desarrollo en la construcción del socialismo, concluimos que la entera década se caracterizó por una dirección voluntarista de la economía, y solo el generoso apoyo de los países socialistas mantuvo en vida la revolución.

Palabras clave: revolución cubana; estrategia de desarrollo; azúcar; voluntarismo.

Abstract. In this work we attempt to demonstrate that the economic difficulties experienced at the beginning of the revolutionary period were rooted initially in a badly-designed development strategy, and later in the implementation of a management system of the economy that dispensed not only with the market, but

CÓMO CITAR: Sovilla, B. (2019). Dificultades y contradicciones en la construcción del socialismo en Cuba al inicio del periodo revolucionario. America Latina en la Historia Económica, 26(3), e983. DoI: 10.18232/alhe.983 
with basic principles of economic calculations, at the hearth of central planning. After analyzing official statistics and the debate that took place in Cuba in the mid 1960s regarding which scheme of economic management is most suitable for constructing a socialist system in a developing country, we conclude that throughout that decade, Cuba's economy was directed on a volunteer basis, and that only the generous support received from other socialist countries kept the revolution alive.

Cuban revolution; development strategy; sugar; voluntarism.

JEL: N16; N36.

Recibido: 14 de abril de 2018

Aceptado: 19 de junio de 2018

Publicado: 11 de enero de 2019

\section{INTRODUCCIÓN}

En este trabajo mostraremos cómo la revolución cubana significó desde su inicio un cambio radical en las relaciones de producción y de propiedad, por eso inevitablemente desembocó en un conflicto frontal con el gobierno estadunidense y en un acercamiento al campo socialista.

Antes de la revolución la economía cubana había perdido el dinamismo que la caracterizó a lo largo de la primera mitad del siglo xx, después de ser la última colonia española en América Latina en lograr la independencia en 1902. Sin embargo, había alcanzado los mayores niveles de desarrollo en el continente latinoamericano, aun evidenciando profundos desequilibrios internos. Según Carmelo Mesa-Lago (2009), en la década de 1950 la economía cubana tenía:

una tasa de crecimiento económico reducida que beneficiaba especialmente al capital y la fuerza laboral empleada y sindicalizada; un sector azucarero básicamente estancado y un sector no-azucarero creciente pero insuficiente para generar crecimiento económico vigoroso; alto desempleo que se agudizaba después de la cosecha azucarera; notable brecha en los indicadores socioeconómicos entre las zonas urbanas y rurales; y fuerte dependencia en la exportación del azúcar, así como en la relación económicocomercial con los Estados Unidos (Mesa-Lago, 2009, p. 42).

Antonio Santamaría (2014) confirma el análisis de Mesa-Lago, pero hace hincapié en la dependencia de la economía cubana de las exportaciones de azúcar y del mercado estadunidense "en 1959 Cuba era una de las economías latinoamericanas con mayor PIB per cápita y la más equitativa [...] también era la única que no había experimentado cambios estructurales tras la crisis de 1930. Como resultado su crecimiento era muy dependiente de la producción azucarera y su exportación a EEUU, que adquiría el $80 \%$ \% (p. 694).

Armando Nova (2008) subraya una consecuencia de esta deformación estructural, es decir, la gran dependencia de las importaciones de alimentos en la década de 1950.

En este contexto, las políticas redistributivas de los primeros años permitieron al gobierno revolucionario fortalecer su base de apoyo entre los sectores sociales más pobres y hasta ese momento discriminados. Obviamente las familias y empresas expropiadas se opusieron y en buena parte migraron hacia Estados Unidos, presionando al gobierno estadunidense a tomar medidas 
para obstaculizar la revolución ${ }^{1} \mathrm{Al}$ incremento del poder de compra de la población no correspondió una mayor oferta de bienes y servicios, lo cual empezó a generar tensiones inflacionarias que inicialmente se lograron controlar con acciones administrativas como el canje de dinero y la introducción de la libreta de abastecimiento. Siendo los precios también controlados por vía administrativa el desequilibrio entre demanda y oferta tenía como manifestación un creciente excedente monetario entre la población.

El fervor revolucionario inicial no siempre propició decisiones económicas racionales. Por ejemplo, el comprensible afán de liberarse del azúcar considerado el símbolo de la explotación colonial e imperialista ${ }^{2}$ determinó un cambio demasiado rápido en la estrategia de desarrollo: ${ }^{3}$ al tratar de diversificar las exportaciones, sin haber creado las condiciones para poder sostenerlo. El reconocimiento de este error llevó al grupo dirigente a un cambio radical en la estrategia de desarrollo centrándola ahora en el incremento de la producción y exportación de azúcar y generar la divisa necesaria para financiar las importaciones. Así surgió el plan para producir 10000000 de toneladas de azúcar en 1970. No obstante, después de haber eliminado el mercado como mecanismo de generación de los incentivos y de redistribución de la producción, en el intento de reorganizar la economía para apoyar esta nueva estrategia, se descuidaron los principios del cálculo económico aplicados por los países socialistas. Este segundo error fue de mayores proporciones y causó una grave crisis económica a finales de la década de 1960, de la cual Cuba se recuperó gracias al generoso apoyo soviético.

De acuerdo con Mesa-Lago $(1981,1994,2009)$ la década de 1960 se subdivide en distintas etapas, según los cambios en la estrategia de desarrollo y el sistema de dirección de la economía adoptados. En este trabajo proponemos una evaluación menos convencional, se hace hincapié en el papel dominante del líder de la revolución en todas las decisiones más importantes. Por ejemplo, no coincidimos con la identificación de una etapa mao-guevarista entre 1966-1970, propuesta inicialmente por Mesa-Lago, y sucesivamente adoptada por la gran mayoría de los cubanólogos 4 (Brundenius, 1984, pp. 53-54; Zimbalist y Brundenius, 1989, p. 123; Lataste, 1968, p. 51; White, 1987, p. 154; Bernardo, 1971, p. 11). $\left.\right|^{5}$ También Santamaría (2014) considera que en el "debate entre socialismo de mercado y modelo chino [...] defendidos por Carlos R. Rodríguez y Ernesto Guevara, Castro [...] optó por el segundo" (p. 698).

\footnotetext{
${ }^{1} \mathrm{El}$ intento de organizar una acción contrarrevolucionaria desde playa Girón para derrotar al gobierno revolucionario y la declaración por parte de Estados Unidos del embargo económico, comercial y financiero hacia la isla, son dos acciones concretas planificadas por el exilio cubano con el apoyo del gobierno estadunidense. Lejos de producir un cambio de gobierno, dieron pie al acercamiento entre Cuba y la Unión Soviética.

${ }^{2}$ Según Antonio Santamaría (2014, p. 694) la dependencia de la economía cubana del azúcar "tuvo su origen en las reformas del imperio español desde la década de 1760, que impusieron un proyecto económico colonial basado en la producción de azúcar con esclavos".

${ }^{3}$ Entendemos por estrategia de desarrollo la definición de los objetivos socio económicos y de sus prioridades, así como la determinación de un programa de acción para alcanzarlos (Rodríguez, 1988, p. 21).

${ }^{4}$ Este término se utiliza en Cuba para indicar a los académicos que desde otros países se dedican al estudio del sistema económico y político cubano. Mesa-Lago (2015) prefiere definirlos cubanistas en lugar de cubanólogos, considerando la carga ideológica en este último.

${ }^{5}$ Son pocos los autores que adoptan otra definición para calificar este periodo, Roca (1977, p. 83) lo denomina economía moral. Es interesante la posición contradictoria de Max Azicri (1988, p. 128), quien primero sostuvo que entre 1966-1970 se aplicó un modelo basado en las ideas económicas de Guevara y dos años más tarde, (1990, p. 19) afirmó lo contrario, o sea que las políticas de esos años no reflejaban las ideas de Guevara, atribuyendo este cambio de opinión al trabajo esclarecedor de Carlos Tablada Pérez (1987).
} 
En realidad, en el periodo 1966-1970 Fidel Castro no trató de aplicar el sistema de dirección propuesto por Ernesto Guevara, ni este se puede confundir con el modelo chino, como veremos más adelante. El mismo Castro denominó nuevo sistema de registro el intento de prescindir de los incentivos materiales y de una planificación organizada de la economía, diferenciándolo tanto del sistema de financiamiento presupuestario, como del cálculo económico, el sistema de dirección aplicado en todo el bloque socialista. En una famosa autocrítica en el Primer Congreso del Partido Comunista Cubano, el líder máximo reconoció que fue una etapa idealista en la cual se tomaron decisiones irracionales que llevaron la economía cubana al borde del precipicio en 1970. Por eso proponemos calificar como fidelista este periodo, que casi nada tuvo que ver con el sistema de financiamiento presupuestario defendido por Ernesto Guevara en el debate económico con Carlos Rafael Rodríguez entre 1963 y 1965 6

\section{EL PERIODO DEMOCRÁTICO-POPULAR (1959-1960)}

En los dos primeros años del periodo revolucionario el nuevo gobierno trató de redistribuir la riqueza nacional y determinar los cambios en las relaciones de propiedad necesarios para ese fin. Las principales medidas tomadas en este periodo fueron las siguientes: un aumento entre 8 y $12 \%$ del salario de los trabajadores de la caña de azúcar, la disminución de los alquileres entre 30 y 50 \% y la reforma tributaria que favorecía algunos sectores de la burguesía local (Vilariño, 1980, p. 83). Por efecto de ellas y de la primera reforma agraria, José Luis Rodríguez (1987, p. 30) estima una redistribución entre 9 y $11 \%$ del ingreso nacional en favor de los campesinos en el periodo 1959-1962, lo cual también se tiene que interpretar como un intento de disminuir las diferencias entre las zonas rurales y urbanas y contener la despoblación del campo, uno de los problemas más graves heredados por el gobierno revolucionario, además de perseguir una mayor justicia social.

El artículo 24 de la Constitución de 1940 que prohibía la nacionalización de la propiedad privada se empezó a modificar desde 1959 y eso permitió la intervención de algunas compañías de teléfono y de las refinadoras de petróleo (Vilariño, 1980, p. 65). Otras modificaciones del mismo artículo 24 permitieron la nacionalización de las propiedades de los cubanos que habían dejado el país y eran acusados de actividad contrarrevolucionaria (O’Connor, 1970, p. 157) mientras que el Ministerio para la Recuperación de los Bienes Malversados empezó la expropiación de las propiedades de la burguesía comprometida con la mala administración de los gobiernos anteriores a 1959 (Rodríguez, 1990, p. 40).

\footnotetext{
${ }^{6}$ A pesar de una firme y pública autocrítica sobre los errores de idealismo de esa etapa, Castro volvió a imponer sus criterios voluntaristas de dirección económica en 1986, cuando abrió el proceso de rectificación de errores y tendencias negativas, conocido como rectificación. Y eso se repitió en 2003 cuando lanzó la batalla de ideas. En ambos casos se impusieron decisiones económicas irracionales atribuyendo erróneamente a Ernesto Guevara su paternidad ideológica, en el intento de encontrar un mayor respaldo entre el pueblo. En realidad, esto demuestra que el máximo líder siempre ha subordinado las decisiones económicas con el fin último de preservar el control político.
} 
Después de la nacionalización completada a finales de 1960 de todas las propiedades estadunidenses en la isla $7^{7}$ y de la casi totalidad de las industrias locales 8 en la primavera de $1961,85 \%$ del valor de la producción industrial provenía del Estado y un año más tarde, 90 \% (O’Connor, 1970, p. 167). Desde el punto de vista de la distribución de la fuerza de trabajo seguían siendo privados, a mitad de 1962, $15 \%$ de la industria, $8 \%$ de los transportes y $45 \%$ del comercio. Sin considerar la agricultura, las únicas propiedades privadas eran un cuarto de las industrias de producción de alimentos, $15 \%$ de las que producían textiles y cuero, los restaurantes de propiedad familiar, las empresas de los servicios y las tiendas minoristas.

Con la declaración de embargd 9 económico hacia la isla por parte del gobierno estadunidense en noviembre de 1960, ya no era posible dejar al sector privado el control de centros vitales de la economía del país, como los bancos y el comercio con el exterior. El 23 de febrero de 1961 se instituyó el sistema bancario centralizado y único del Estado, el Banco Nacional de Cuba, mientras que en julio de 1960 se había constituido el Ministerio del Comercio Exterior como único centro autorizado para administrar las operaciones comerciales con el exterior.

Esta primera fase concluyó en abril de 1961, con la proclamación por parte del líder máximo del carácter socialista de la Revolución.

\section{LA REFORMA AGRARIA}

Antes de la revolución, la economía cubana se caracterizaba por desequilibrios estructurales y una extrema dependencia, siendo monoexportadora de azúcar y multimportadora de productos acabados de Estados Unidos, por tanto, la neutralización del latifundio privado era un paso necesario para llegar a una real autodeterminación que representaba un objetivo fundamental para el gobierno revolucionario. El $9.4 \%$ de los propietarios poseía $73.3 \%$ de la tierra, mientras que $66.1 \%$ sólo poseía 7.4 \% (Rodríguez, 1987, p. 24). Alrededor de una cuarta parte de la superficie agrícola se destinaba al cultivo de la caña de azúcar, aunque apenas 54 \% venía efectivamente cultivado. En 1958 el valor agregado creado por la caña era $36.5 \%$ de todo lo que generaba el sector agrícola, y con el arroz, el tabaco y el café llegaba a $53.3 \%$; con un aporte de la ganadería de $32.7 \%$, todos los demás productos agrícolas contribuían sólo 14 \% de la producción total (Agrupación Católica Universitaria, 1970). Parece entonces oportuno afirmar que "Cuba era un país agrícola sin un desarrollo agrícola” (Rodríguez, 1987, p. 24).

\footnotetext{
${ }^{7}$ En abril de 1960 se intervinieron los latifundios de la United Fruit Company y en junio fue el turno de las tres compañías estadunidenses que rechazaron refinar el petróleo crudo soviético. En agosto se nacionalizaron otras importantes empresas estadunidenses (Cuban Electric Company, North American Sugar Industries, Moa Bay Mining Company, West Indies Sugar Company, Bangor Punta Corporation) y en octubre, después de la declaración del embargo, todas las que quedaban (Rodríguez, 1990, pp. 40-41; Kirby Jones, 1988, p. 71).

${ }^{8}$ La ley 78 de febrero 1959 instituyó el ministerio para la recuperación de bienes malversados. La primera importante nacionalización fue la de la Cuban Telephone Company, a la cual siguió inmediatamente después la primera reforma agraria con la intervención de los grandes latifundios. En octubre se nacionalizaron los bancos y las grandes empresas locales, luego las casas y las tiendas (Rodríguez, 1990, pp. 40-41).

${ }^{9}$ En octubre de 1960, en respuesta a las expropiaciones del gobierno cubano de las propiedades de ciudadanos estadunidenses en la isla, Estados Unidos decretó un embargo económico, comercial y financiero en contra de Cuba. A pesar de haber sido condenado por la Asamblea General de las Naciones Unidas todos los años con amplias mayorías en el último cuarto de siglo xx, el embargo (que el gobierno cubano califica como bloqueo) sigue vigente. Sólo a finales de 2014 empezó un acercamiento entre los dos gobiernos que desembocó en la visita del presidente estadunidense Barack Obama a la isla en 2016, sin embargo, con el triunfo electoral de Donald Trump las relaciones han vuelto a enfriarse.
} 
Por tal razón, la medida que mayores repercusiones tuvo en la esfera socioeconómica, además de permitir entender cómo las intenciones del gobierno revolucionario irían más allá de una política puramente redistributiva, fue la primera reforma agraria del 13 de mayo de 1959. La reforma fue implantada por el Instituto Nacional para la Reforma Agraria (en adelante INRA), creado precisamente para ello. Se estatalizaron los grandes latifundios privados y una parte de la tierra se redistribuyó a los trabajadores agrícolas de las grandes plantaciones, y a los que cultivaban parcelas inferiores a las 26 hectáreas (fueran o no propietarios) se les entregó o garantizó su propiedad 10 El artículo 1 prohibía las propiedades mayores de 402 hectáreas y al mismo tiempo establecía las excepciones, o sea las tierras cuyo rendimiento era $50 \%$ mayor que el promedio nacional. Además de la mediana propiedad ${ }^{11}$ no fueron nacionalizadas las grandes propiedades muy productivas. La presencia de elevadas economías de escala en algunas producciones como el arroz, la caña de azúcar y el ganado, sugería no subdividir el latifundio y por eso se crearon las cooperativas agrícolas. Sucesivamente, con la segunda reforma agraria (en 1963) y la política de la persuasión hacia los pequeños propietarios (1967-1975), estas incluyeron las parcelas medianas y pequeñas respectivamente. Las decisiones de política agraria se centralizaron en un solo organismo, el INRA, que se ocupaba también de reorganizar el sistema distributivo, donde se vio desde el inicio un sesgo en contra de la intermediación privada $\sqrt{12} \mathrm{Al}$ interior de este instituto, se creó un departamento para la industrialización, por la gran compenetración entre el sector industrial y agrícola, sobre todo en la producción de azúcar.

Por tanto, con la primera reforma agraria 40 \% de la tierra pasó al Estado y aumentaron también los pequeños propietarios. El rompimiento del latifundio privado y la distribución de la tierra a los que la trabajaban sin ser propietarios (braceros, locatarios, aparceros y ocupantes abusivos), no era más que la aplicación del artículo 90 de la Constitución de 1940 en relación con el latifundio y de la ley número 3 de la sierra Maestra (Martínez, 1978) promulgada desde la clandestinidad por el líder guerrillero Castro unos meses antes del triunfo de la revolución.

A los propietarios medianos les tocaba, después de la reforma, sólo $23.2 \%$ de la tierra (Rodríguez, 1987, p. 27), sin embargo, participaban abundantemente en producciones importantes como el azúcar (producían entre 15 y 20 \% del total) y en la ganadería; la segunda reforma agraria en septiembre 1963 se propuso eliminar esta clase social, los kulaki locales, en favor del sector estatal que en sólo cuatro años llegó a ser propietario de dos terceras partes de la superficie agrícola del país.

En 1961 se había terminado un proceso de estatalización en el cual la primera reforma agraria era el paso más delicado, acompañado por una importante redistribución de la riqueza y un aumento, sobre todo para los sectores más pobres, del poder de compra, lo que justificó un apoyo popular indiscutible en favor del gobierno revolucionario.

\footnotetext{
${ }^{10}$ Según José Acosta (1973, p. 62): “los campesinos que trabajaban tierras con una extensión superior a 26 hectáreas, pero menor que 67, recibían tierras hasta completar esta extensión”. Peter Gey (1987, p. 92) afirma que esta medida no se aplicó.

${ }^{11}$ Se consideraban grandes propiedades las parcelas superiores a 402 hectáreas, medianas propiedades las que estaban entre 67 y 402 hectáreas y pequeñas propiedades las que tenían menos de 67 hectáreas.

${ }^{12}$ Se creó una red de empresas estatales de acopio, para centralizar la recolección de productos agrícolas del sector privado; también se constituyeron las tiendas del pueblo para la distribución de productos finales.
} 
Después de haber modificado las relaciones de fuerza entre las clases sociales y estatalizado gran parte de la economía, había que pasar a la fase más difícil, reorganizando el aparato productivo para que el aumento del poder de compra de las clases sociales más pobres, se convirtiera en un efectivo y duradero mayor bienestar.

Hasta finales de 1961 no se observaron graves restricciones por el lado de la oferta. Las nacionalizaciones y otras medidas como la disminución de importaciones de bienes de lujo contribuyeron a generar un cuadro de estabilidad macroeconómica, tanto en las finanzas públicas como en el sector exterior, absorbiendo también las presiones inflacionarias.

Pero ya a principio de 1961 se empezaron a manifestar los primeros problemas. En la conferencia nacional sobre la producción en La Habana en agosto de 1961, los máximos representantes del gobierno a pesar de reconocer las dificultades, evidenciaron subestimarlas (Boorstein, 1968, p. 91). También los representantes más preparados y pragmáticos hicieron previsiones totalmente irrealistas sobre el futuro de la economía (Ritter, 1974, p. 137), mientras que unos meses más tarde (en marzo de 1963) fue necesario introducir la distribución racionada de algunos bienes de primera necesidad, todavía en vigor, ya que por obvias razones el gobierno rechazó utilizar el mecanismo de precios para neutralizar el exceso de demanda que se había determinado.

\section{EL PROBLEMA AGRARIO}

Con la primera reforma agraria el país había sido dividido en 28 zonas de desarrollo agrícola. En cada una de ellas había un administrador del INRA, normalmente un ex oficial del ejército rebelde, que dirigía la aplicación de la reforma agraria.

En las tierras que pasaron al Estado se formaron las cooperativas agrícolas, las haciendas directamente administradas y las cooperativas de la caña de azúcar. Las primeras se parecían a los kolkhoð ${ }^{13}$ soviéticos y según Conchol (véase en Ritter, 1974, pp. 73-74) “de las cooperativas sólo tenían el nombre y el deseo". Dentro de ellas no había ninguna forma de control desde abajo sobre la dirección (compuesta por funcionarios del INRA), así como nunca entró en vigor la anunciada repartición de las ganancias, por la total ausencia de los mecanismos contables más elementales. En las haciendas directamente administradas (que existían sobre todo en la rama arrocera y en la ganadería) los márgenes de autonomía eran aún menores y tanto los administradores como los obreros agrícolas dependían directamente del INRA. También en el caso de la caña, así como en el arroz y la ganadería, por el carácter estratégico de estas ramas, no era aconsejable dejarlas a productores particulares o fragmentar las parcelas: se formaron así las cooperativas de la caña, estructuras híbridas según Ritter (1974), donde había una administración elegida por el INRA y un consejo directivo elegido desde la base.

\footnotetext{
${ }^{13}$ Unos años después de la toma del poder por parte de los bolcheviques (1917), el gobierno revolucionario soviético empezó la reforma agraria, que se basaba en la creación de dos tipos de empresas agrícolas socialistas: el sovkhoz, considerada la forma superior de producción, era una empresa estatal en la cual los trabajadores recibían un sueldo fijo, siendo empleados estatales. La otra forma de producción socialista se denominaba kolkhoz, y eran cooperativas que surgían de la unión de pequeños propietarios terreros.
} 
La reforma agraria se llevó a cabo en sólo 18 meses (se preveían dos años) y dos medidas tomadas a principio de 1961 marcan el fin de esta primera fase de la revolución. Se trata: $a$ ) de la fusión ${ }^{14}$ entre cooperativas agrícolas y haciendas directamente administradas que dio origen a las haciendas agrícolas estatales, las granjas (Ritter, 1974, p. 75) que Castro consideraba la forma superior de producción ${ }^{15}$ Muy parecidas a los sovkhoz soviéticos y con un tamaño promedio aún mayor, fueron las unidades productivas en las cuales ha apoyado el desarrollo agrícola del país por más de 30 años. En 1962 también las cooperativas cañeras se transformaron en granjas (Boorstein, 1968, p. 45). Estas enormes parcelas cuyo tamaño promedio era de 9000 hectáreas en mayo de 1961 empleaban 96500 trabajadores (Ritter, 1974, p. 75). Respecto a la cooperativa la granja era más extensa y tenía una cantidad de tierra por trabajador mucho mayor, con una administración además más centralizada. $b$ ) La unión de los pequeños propietarios con propiedades individuales de menos de 67 hectáreas en la asociación nacional de agricultores pequeños. Esta organización inicialmente tenía una finalidad exclusivamente económica, ${ }^{16}$ pero en un segundo momento se convirtió en un brazo del gobierno (Domínguez, 1978, p. 447), un organismo de presión ideológica cuyo objetivo era convencer a los pequeños campesinos a apoyar los cambios en la política agraria decididos por el gobierno, llegando a promover su incorporación en las haciendas estatales.

En los meses que siguieron a la reforma agraria hubo una transferencia de poder decisional de los dirigentes del INRA hacia la administración de la granja, que no se debe interpretar como el prevalecer de una política descentralizadora después del centralismo del primer periodo, sino como una necesidad debida a la incapacidad, por parte del centro, de dirigir directamente el sector agrícola, por la cantidad de tierra estatalizada en tan poco tiempo.

Como consecuencia, hubo un periodo de administración totalmente improvisado, por la libre, según Michel Gutelman (1969). Ernesto Che Guevara lo calificó como guerrillerismo administrativo (véase en Boorstein, 1968, p. 48) para indicar que los métodos de gestión y las mismas personas encargadas habían sido transferidos de la guerrilla. Según un agudo observador de aquel periodo "la descentralización de facto se impuso. El INRA no tenía la organización y los conocimientos para ejercer una dirección sistemática y controlar las cooperativas y las haciendas estatales. Había una centralización de las decisiones sobre problemas específicos, a menudo sobre detalles" (Boorstein, 1968, p. 49). Gutelman (1969) prefiere hablar de planificación sectorializada en lugar de descentralización, por la total ausencia de coordinación a nivel local.

Los problemas de esta sectorialización aparecieron pronto. Todo el esfuerzo organizativo que había empezado en 1962 y sigue todavía [en 1967] tiene como objetivo instituir un sistema de dirección de la agricultura no menos centralizado, como a me-

\footnotetext{
${ }^{14}$ Acosta (1973, p. 67) resume las causas que han movido en esta dirección: la cooperativa disponía de poca fuerza de trabajo, sobre todo faltaban técnicos, lo cual justificaba la tendencia a la centralización; además los obreros agrícolas tenían poca propensión a decidir autónomamente y dada la importancia de la ganadería para el consumo interno, era riesgoso otorgar una autonomía que podía quedarse en un plan muy formal.

15 “en las cooperativas agrícolas el trabajador trabaja para sí mismo mientras que en las factorías del pueblo trabaja para la nación. En las cooperativas hay dos tipos de trabajadores, los socios que son dueños y los asalariados y el segundo grupo viene explotado por el primero, que rápidamente abandona su status de dueño y se convierte en semi explotador" (citado en Mesa-Lago, 1968, p. 33).

${ }^{16}$ La Asociación Nacional de Agricultores Pequeños organizaba la entrega de tractores, fertilizante, la otorgación de préstamos, la determinación de los precios de los productos, funciones que sucesivamente pasaron al INRA y al Banco Nacional de Cuba.
} 
nudo se piensa, sino menos sectorializado. La lucha contra la sectorialización implica obviamente cierta descentralización de las decisiones, porque la sectorialización es en parte el resultado del excesivo centralismo. Los factores que movían hacia la sectorialización todavía no han desaparecido: los cuadros disponibles, aún en rápido aumento, son todavía insuficientes; el bloqueo económico todavía no ha sido quitado; las decisiones de política económica tienen en buena medida un carácter político porque la lucha de clase en Cuba no ha terminado todavía [...] En estas condiciones el problema del centralismo sectorializado se puede resolver solo lentamente, mientras se vayan aliviando las constricciones que pesan sobre la economía cubana (Gutelman, 1969, p. 100).

Gutelman ya tenía muy claro, a finales de la década de 1960, lo que se reveló ser una característica permanente de la conducción de la economía en Cuba, es decir, la concentración del esfuerzo en algunas ramas o sectores, en perjuicio de otros, con las pérdidas que normalmente superan los beneficios, en ausencia de una efectiva planificación central y por tanto al margen de una visión sistémica de la economía, lo cual entraba en total contradicción con el modelo socialista soviético que se difundió también en Europa Oriental. Sin embargo los años siguientes han demostrado que las causas no eran las que mencionada Gutelman -los efectos negativos del embargo estadunidense fueron siempre más compensados por el creciente apoyo soviético, la lucha de clase dejó de representar un peligro para el gobierno 17 y los cuadros técnicos aumentaron notablemente-, siendo más estructurales. Esa visión asistémica y sectorial de la economía mencionada por Gutelman fue una constante en la dirección de la economía por lo menos hasta que el ejercicio del poder se concentró en Fidel Castro.

\section{PRIMERAS DIFICULTADES Y ERRORES}

El principal obstáculo a la planificación económica después de los cambios importantes en las relaciones de propiedad y la eliminación del mercado, tenía que ver con la construcción de un aparato estadístico que entregara datos confiables sobre la actividad económica, indispensables para poder planificar. La huida del personal técnico y administrativo había dejado un vacío preocupante, siendo imposible reemplazarlo inmediatamente con personal local. A veces se entregaba a jóvenes revolucionarios la responsabilidad en la conducción de ingenios azucarero ${ }^{18}$ o de plantas productivas tecnológicamente avanzadas, lo cual llevaba a ese guerrillerismo administrativo que el mismo Guevara consideraba un gran obstáculo en el camino para el desarrollo.

Además, se había cometido un error fundamental, trazando una estrategia de desarrollo demasiado ambiciosa e irrealista, que buscaba una rápida diversificación en la producción agrícola y un acelerado proceso de industrialización, lo que ponía aún más en evidencia la escasa preparación

\footnotetext{
${ }^{17} \mathrm{Al}$ inicio del periodo revolucionario hubo dos intentos de derrocar al régimen con la fuerza: la invasión de Bahía de Cochinos y el levantamiento armado en el Escambray. El primero fue neutralizado en tres días, mientras que las acciones en contra del gobierno castrista en el Escambray duraron algunos años, terminando en 1965.

${ }^{18} \mathrm{El}$ sociólogo e investigador Juan Valdés Paz aclaró que al inicio de la revolución le fue asignada la dirección de un central azucarero, aun siendo joven y sin experiencia ninguna en este campo y que lo mismo les pasó a otros jóvenes revolucionarios inexpertos y sin preparación técnica para desenvolverse en las tareas asignadas. (J. Valdés, comunicación personal con el autor, 1994).
} 
técnica, la falta de experiencia administrativa de los jóvenes dirigentes de empresas apenas nacionalizadas y la carencia de información estadística que ponían serias limitaciones a la actividad de planificación.

Entre 1961-1963 se buscó diversificar la producción agrícola y un desarrollo industrial que permitiera sustituir las importaciones. Los expertos de la Comisión Económica para América Latina y el Caribe apoyaban esta estrategia y algunas evidencias también históricas parecían aconsejar a emprender el nuevo camino 19

Los resultados fueron indudablemente catastróficos: la reducción de la superficie destinada al azúcar que Ritter (1974, p. 179) estimó en 10 \% de la superficie total, ${ }^{20}$ los graves problemas organizativos del INRA en gran parte producto de la misma estrategia, determinaron una gran caída en la producción en 1962 y 1963 . La zafra de 1963 fue la mitad de la de 1961 y solamente gracias al precio elevado del azúcar en el mercado mundial, la reducción de los ingresos en el mismo periodo fue apenas de $10 \%$. En 1962-1963 en lugar de una diversificación en la producción, sobrevino una fuerte reducción de los principales productos de exportación, como el tabaco y el azúcar, aunque como consecuencia de la reducción de las otras exportaciones, el peso del azúcar sobre el total pasó de $78 \%$ en 1959-1960 a $85 \%$ en 1962-1963, a pesar de la caída en la producción. La disminución de los rendimientos de los cultivos fue notable (Ritter, 1974, p. 147).

En los dos años que siguieron a la primera reforma agraria a pesar de los enormes problemas de reorganización de la economía, las consecuencias del embargo económico estadunidense y la invasión militar de mercenarios cubanos en playa Girón, la producción agrícola mostró incrementos inesperados, pero después del cambio en la estrategia de desarrollo en 1961 la caída fue vertical 21

En la industria las nacionalizaciones fueron rápidas -se efectuaron en tres meses- con un fuerte impacto sobre la economía, e incluyó 85 \% del sector en 1961. Donde era posible se estimularon los consolidados (uniones horizontales entre empresas), para aprovechar con mucho realismo los métodos contables y administrativos muy avanzados aplicados por las grandes filiales de empresas estadunidenses. Esta concentración también permitía atenuar las fallas técnico-administrativas por la salida del país de gran parte del personal especializado, no fácilmente sustituible en el corto plazo. En este contexto se abrió camino en la industria y en el comercio exterior, la idea guevarista del sistema de financiamiento presupuestario, como alternativa a la autogestión financiera aplicada en los países de Europa Oriental.

\footnotetext{
${ }^{19}$ Por ejemplo, se observó que la URSS había privilegiado con cierto éxito la industrialización en la década de 1920 , también gracias a una contracción del consumo y una acumulación generada en el sector agrícola (en Cuba no se discutió mucho sobre el financiamiento de esta estrategia), reintroduciendo el mercado con la nueva política económica aprobada por Lenin después del caos de la etapa inicial denominada comunismo de guerra.

En los primeros años de la década de 1960 el mercado del azúcar no ofrecía buenas perspectivas y los dirigentes revolucionarios manifestaron una visceral aversión hacia ese producto que era considerado el símbolo de la explotación imperialista. Sin embargo, en la presentación del plan económico para 1962 el entonces ministro de Economía Regino Boti (1961) todavía declaraba el objetivo de "asegurar un nivel alto y estable en la producción de azúcar" y al mismo tiempo disminuir el peso del azúcar sobre las exportaciones totales, aumentando las exportaciones no azucareras.

${ }^{20}$ Según Rodríguez (1990, p. 96), en agosto de 1961 la superficie cultivada para la producción de azúcar se redujo a 130000 hectáreas. En las palabras de Carlos Rafael Rodríguez, uno de los principales dirigentes revolucionarios y destacado economista, se cometió el error de menospreciar la caña como si eso fuera indispensable para diversificar la agricultura, transformando cada granja en un mosaico de cultivos.

${ }^{21}$ También James O’Connor (1970, p. 223) reconoció como un grave error por parte del InRA introducir “casi de un día para otro un programa de diversificación de los cultivos en gran escala”.
} 
En 1963 estaba claro que cada intento de planificación y la misma elección de un sistema de dirección de la economía, necesitaban que se volviera a replantear una estrategia de desarrollo alternativa ${ }^{22}$ Ernesto Guevara, ministro de Industria, empezó una fuerte autocrítica. Denunció (1964) que la elección de las inversiones no era sabiamente definida, ya que se buscaba sustituir las importaciones de los bienes más costosos, sin dirigir la atención hacia los costos y las reales condiciones de producción. Se buscaba producir sustitutos de bienes finales importados, y no se entendía que se necesitaba comprar materias primas importadas para producirlos.

En los primeros años no se observaron los aumentos esperados de productividad ni de capacidad productiva. Según Ritter (1974, p. 154), las inversiones netas entre 1961-1963 eran cercanas a cero o negativas, lo cual significa que los nuevos planes de inversión ocurrían mientras se deterioraba la capacidad productiva instalada. Inicialmente se trató de importar piezas de repuesto del área capitalista, pero al observar que no se lograba detener el deterioro de las maquinarias, se importaron ex novo de los países del este de Europa.

Consideramos que el periodo 1961-1963 fue efectivamente una fase de fuerte idealismo a pesar que la cubanología y fuentes gubernamentales apliquen esta definición a la segunda mitad de los sesentas, y no a este periodo, malinterpretando así las características de toda la década.

El fracaso de la primera estrategia de desarrollo era claro en 1963: no se había empezado un proceso de industrialización y en la agricultura se registraba el colapso de muchas producciones, en lugar de un proceso de diversificación como se había programado.

Los importantes déficits comerciales, los acuerdos con China y la URSS para estabilizar en niveles razonables (superiores al costo de producción) el precio del azúcar y las cantidades que se comprometían a comprar, además del contenido bajo de importaciones del azúcar y del tabaco, movieron hacia un cambio radical en la estrategia de desarrollo. Según Ritter (1974, p. 183), se pasó de la sucrofobia a la sucrofilia, al establecer una estrategia de desarrollo más ortodoxa con el azúcar en lugar de la industria pesada. La idea esta vez más realista era que los ingresos altos y estables de las exportaciones azucareras habrían permitido algunos años después financiar un desarrollo industrial. También en esta ocasión, al terminar el periodo, los resultados eran muy negativos, pero las causas ahora radicaban en el sistema de dirección establecido.

\section{LA PLANIFICACIÓN Y EL DEBATE SOBRE EL SISTEMA DE DIRECGIÓN DE LA ECONOMÍA}

Con la asesoría de expertos planificadores provenientes de Checoslovaquia que llegaron a la isla en diciembre de 1960, y de economistas prestigiosos como Michael Kalecki y Charles Bettelheim, se introdujo en Cuba el sistema de planificación checo. 23

\footnotetext{
${ }^{22}$ Cabe precisar que el desequilibrio monetario y financiero que obligó a introducir el racionamiento a principios de 1962, había empezado mucho antes. Según datos de Rodríguez (1990, p. 89), la masa monetaria en la población pasó de “425000 000 de pesos en 1958 a 475000000 en 1959 y a 910000000 en 1960”. Este primer desequilibrio fue absorbido con un mayor uso de la capacidad productiva ociosa en la producción de bienes de consumo, pero en junio de 1961 había 1187 millones de pesos en circulación y sólo con el canje del dinero de agosto hubo un retiro de 497600000 de pesos que eliminó el desequilibrio mencionado. Sin embargo, este se volvió a presentar unos meses después, lo cual indujo a introducir el racionamiento en marzo de 1962. Lo que sucedió en ese periodo es una prueba que cuando aparece una situación inflacionaria se necesitan, también en una economía planificada, medidas estructurales, que determinen un incremento en la productividad de los factores y en la oferta.

23 Alban Lataste (1968, p. 61) aclara que el plan anual para la economía nacional estaba dividido en cinco secciones o categorías fundamentales: producción y asignación de recursos, trabajo y salarios, finanza y costos e inversión. El estudioso menciona que tomando en cuenta las orientaciones del gobierno, se establecen las metas de producción para
} 
El gobierno establecía los objetivos principales (por ejemplo, la tasa de crecimiento del producto social global ${ }^{24}$ la división del producto entre consumo e inversión, el utilizo de la capacidad productiva). Luego, estos indicadores generales pasaban a la junta central de planificación que los subdividía en distintos grupos, donde se definían las cifras de control. Estas se referían a un conjunto de indicadores que a su vez se subdividían en otros subconjuntos (por ejemplo, una cifra de control era la producción, que comprendía la producción en valor, la producción física, la capacidad productiva, los índices o normas relevantes). ${ }^{25}$ Las cifras de control se transmitían a los administradores de las empresas que las discutían en las asambleas con los trabajadores luego las volvían a subir hacia arriba para que recorrieran el camino contrario. El plan regresaba así al Consejo de Ministros, de donde había salido, que aprobándolo lo convertía en ley del Estado. En un segundo momento la Junta Central de Planeación volvía a transmitir las cifras a las empresas, esta vez ya no eran de control, sino directivas, o sea obligatorias 26

En realidad, había problemas muy concretos que obstaculizaban los planificadores. Por ejemplo, hasta que el INRA no pudo hacer el inventario de los bienes de propiedad estatal (inicio de 1963), no se conocía la cantidad de capital a disposición de las empresas agrícolas y sólo en 1964 se pudo calcular el capital fijo en la industria, en el transporte y otros sectores. No sorprende por tanto la afirmación de O’Connor (1970, p. 260), según la cual hasta 1964 la Junta Central de Planeación no podía medir las tasas de depreciación del capital fijo para definir los valores eficientes de inversión. También había problemas con los datos en el área de costos y en la administración financiera, por ejemplo, los gastos se limitaban al pago de salarios.

Sin embargo, los dos obstáculos principales para la planificación eran constituidos por una estrategia de desarrollo demasiado ambiciosa comentada en el párrafo anterior, y la aplicación simultánea de dos sistemas de dirección de la economía entre ellos incompatibles: el cálculo económico y el sistema de financiamiento presupuestario.

Este sistema de financiamiento se empezó a aplicar en 1960 en la industria, y en parte del comercio exterior, después de la estatalización de las empresas estadunidenses y nacionales. La falta de cuadros técnicos y administrativos indujo a formar un fondo centralizado para financiar la zafra que más tarde se incorporó en el presupuesto público para financiar todas las empresas industriales.

Se pensaba que para suplir la falta de personal directivo y aprovechar el avanzado sistema de contabilidad de las modernas empresas estadunidenses en la isla, era conveniente mantener grandes unidades empresariales, por eso se buscó la fusión de las mismas en combinados.

En el sistema de financiamiento presupuestario las empresas actuaban como unidades operativas de una empresa estatal superior y entre ellas no había contratos de compraventa, que eran sustituidos por asignaciones administrativas (un organismo de la administración central suminis-

sectores y ramas de actividad, luego se hacen compatibles estas metas con los balances materiales estableciendo las fuentes y destinos de los productos principales. Determinada la proporción entre las metas de producción y los recursos materiales se calcula la inversión para completar las metas y los flujos financieros necesarios.

${ }^{24} \mathrm{El}$ producto social global era el indicador utilizado en la contabilidad de los sistemas económicos planificados. Mide el valor bruto de la producción y no utiliza el método del valor agregado, por tanto, los bienes intermedios se valoran dos o más veces. Por otro lado, excluye los servicios no materiales, como la educación.

${ }^{25}$ Las cifras de control se referían a ocho agregados: la producción, la oferta, la distribución, la inversión, la fuerza de trabajo, los costos, las finanzas y los nuevos productos.

${ }^{26}$ Según O’Connor (1970, p. 257), el proceso de planificación sólo aparentemente es muy democrático, en realidad la participación de los trabajadores en su elaboración es muy limitada. 
traba a las empresas los recursos necesarios) ${ }^{27}$ Cada empresa tenía dos cuentas en el Banco Nacional de Cuba, en una depositaba los ingresos y en la otra se podía financiar para sufragar sus gastos. Junto con el Ministerio de Hacienda, el Banco Nacional llevaba controles rigurosos sobre los costos de las empresas, por eso era importante mantener el sistema de contabilidad avanzado de las empresas estadunidenses transfiriéndolo a los combinados. El dinero tenía que perder sus funciones de reserva de valor y de medio de pago, manteniendo sólo la de unidad de cuenta para medir las transacciones. El objetivo era llegar a prescindir totalmente del dinero en la fase superior comunista, hacia la cual el sistema socialista tenía que evolucionar. Sólo en esta fase final los estímulos materiales podían desaparecer, ya que con la formación del hombre nuevo 28 la eficiencia económica dependía de la conciencia comunista, en la cual el esfuerzo personal se justificaba en valores superiores al materialismo capitalista, como la solidaridad interpersonal y la fraternidad entre los pueblos.

En el cálculo económico las empresas estatales socialistas deben autofinanciarse, o sea cubrir sus gastos con los ingresos generados por su actividad económica, sin poder recurrir al presupuesto central. Se comportaban por tanto como empresas capitalistas, buscando ganancias, relaciones horizontales con otras empresas y el crédito bancario cuando era necesario. La fuerza de trabajo venía motivada con estímulos materiales, como en el sistema capitalista. Este sistema de dirección de la economía se aplicaba en la URSS y su principal sostenedor en Cuba era Carlos Rafael Rodríguez, ex dirigente del Partido Socialista Popular y filo soviético, quien fue nombrado director del INRA en 1963 y trató de insertar el cálculo económico en las granjas sin mucho éxito. El mismo Fidel Castro (1990, p. 103) afirmó que el sistema del cálculo económico "funcionó de manera parcial y muy limitada”. Según Lataste (1968, p. 37), las empresas que adoptaban el cálculo económico eran las que más violaban la ley 1007 recurriendo a la muleta del presupuesto central.

En 1965, Ernesto Guevara, quien había introducido en la industria el sistema de financiamiento presupuestario se fue a exportar la lucha guerrillera primero a Congo y el año siguiente a Bolivia. Rodríguez entregó a Castro la dirección del InRa que empezó el desmantelamiento de ambos sistemas de dirección de la economía. En las mismas palabras de Castro (1990, p. 104) al Primer Congreso del Partido Comunista Cubano en 1975, no había un único sistema de dirección de la economía y en esa circunstancia "tomamos la decisión menos correcta”, inventando un nuevo procedimiento e interpretando con ideología el marxismo "quisimos establecer nuestros propios métodos", al establecer un sistema de dirección de la economía que se alejaba del cálculo económico aplicado en los países socialistas y del sistema de financiamiento presupuestario que se había empezado a aplicar en Cuba, basado en la eliminación de las categorías mercantiles y la supresión de pagos y cobros entre empresas estatales.

\footnotetext{
${ }^{27}$ Luis Álvarez (1964), secretario de Hacienda y partidario del sistema de financiamiento presupuestario, afirmó que las relaciones entre empresas estatales no debían ser intercambios entre mercancías y dinero, sino una entrega y recepción de productos de acuerdo con un plan.

${ }^{28}$ Según Ernesto Guevara (1962), para llegar al socialismo y luego al comunismo era necesario construir el hombre nuevo, o sea un ser humano solidario, que no actúa impulsado por el egoísmo, sino por un ideal superior de bienestar colectivo. Su esfuerzo cotidiano debería provenir de estímulos morales y no materiales.
} 
EL EXPERIMENTO CUBANO EN LA CONSTRUCGIÓN DEL COMUNISMO: DESORDEN ECONÓMICO Y CRISIS

La segunda mitad de la década de 1960 ha sido calificada por Mesa-Lago y la mayoría de los cubanólogos como etapa mao-guevarista. En realidad, las propuestas económicas de Ernesto Guevara buscaban un camino alternativo en la construcción del socialismo, tanto al modelo soviético como al modelo chino, y no fueron instauradas en este periodo de idealismo extremo, donde Castro mantuvo todas las decisiones principales (de allí la connotación de etapa fidelista).

Los principales cambios introducidos fueron los siguientes: la Junta Central de Planeación perdió poder y la dirección de la economía se centralizó en la figura del comandante en jefe. Se multiplicaron los planes especiales (también denominados en la jerga popular planes Fidel) cuyos responsables eran nombrados directamente por el mismo Castro, quien también había asumido, como se dijo, la dirección del InRA. En la autocrítica del Primer Congreso del Primer Congreso del Partido Comunista Cubano, Castro (1990, p. 104) afirmó que se suprimió el presupuesto público (el último se aprobó en 1967), las empresas estatales dejaron de realizar entre ellas cobros y pagos, se abrogaron las escalas salariales introducidas en 1961 y las normas y se introdujo el horario por conciencia, con horas extra voluntarias y gratuitas. Las empresas tenían sólo objetivos de producción definidos en términos físicos, sin ningún control sobre los costos de producción. Castro definió este sistema de dirección el nuevo sistema de registro económico, reconociendo que fue una decisión equivocada y una interpretación idealista del marxismo.

Ninguna de las reformas señaladas en este periodo fue inspirada por Ernesto Guevara. En el sistema de financiamiento presupuestario la Junta Central de Planeación desempeñó un papel central, el control sobre los costos tiene que ser muy rígido y escrupuloso y también sobre el presupuesto central. De acuerdo con Nelson Valdés (1979), el modelo establecido entre 19661970 fue ideado por Castro y es totalmente distinto del sistema elaborado por Guevara, razón por la cual puede denominarse modelo fidelista.

Los resultados de la aplicación del modelo fidelista fueron indiscutiblemente desastrosos. Según estimaciones de Mesa-Lago (1968) derivadas de estadísticas oficiales cubanas (Banco Nacional de Cuba, 1977) y de Brundenius (1984), el PIB per cápita tuvo una contracción de $9.6 \%$ entre 1965 y 1971. Según Rodríguez (1988) el excedente monetario pasó de 574000000 de pesos en 1965 a 3478000000 de pesos en 1971. El mismo Castro (1990, p. 105) reconoció que esto creó condiciones que propiciaron el ausentismo y la indisciplina laboral. Algunos productos del campo como el maíz desaparecieron, la producción de tubérculos importantes en la dieta alimentaria cubana como la yuca, el ñame, el boniato y la malanga, se redujo 90 \% entre 1957 y 1970 (Ritter, 1974, p. 191). La producción de frutas (plátano, piña) también sufrió una caída vertical y la de leche cayó $30 \%$, también la de carne de puerco y de pollo se redujeron considerablemente.

Todo esto a pesar del enorme esfuerzo inversionista realizado en el periodo en el sector agropecuario: por ejemplo, aumentó $30 \%$ la superficie cultivada entre 1965 y 1970, y se multiplicó por dos el número de tractores entre 1968 y 1970.

La estrategia de desarrollo diseñada para este periodo se centraba en concentrar los esfuerzos en la producción de azúcar, con el objetivo de producir 10000000 de toneladas en 1970. Según Santamaría (1994, p. 111), "[e]ntre 1959-1970 los lideres revolucionarios intentarán instaurar una política de industrialización y diversificación económica marcadamente antiazucarera”, pero es evidente que con el cambio en la estrategia de desarrollo en 1964 termina el intento de diversificar las exportaciones y se vuelve a considerar la producción azucarera como la fuente imprescindible de divisa que había sido hasta 1959. De esta manera se pretendía promover las exportaciones y 
generar a través del sector exterior el financiamiento de la inversión interna. La estrategia fracasó: entre 1965-1970 se planificó la producción de 47000000 de toneladas de azúcar, pero se produjeron sólo 35100 000. Esto significó el incumplimiento en la entrega del producto a la URSS por una cantidad casi equivalente a la diferencia entre producción real y planificada (se entregaron a los soviéticos 12100000 toneladas, la mitad de lo acordado para este periodo). Las exportaciones a la URSS disminuyeron entre 1966-1969 a una tasa anual de 5.4 \% (cálculos con base en Rodríguez, 1992, p. 63). El déficit comercial con la URSS entre 1968-1972 fue siempre mayor que el total de las exportaciones hacia ese país.

Aun criticando la herejía cubana (Karol, 1970) ${ }^{29}$ los soviéticos se encargaron de asegurar la sobrevivencia de la revolución. En 1969 las ventas de azúcar en el mercado mundial fueron de 2180000 toneladas, mayores que las exportaciones a la URSS (1 350000 toneladas). Estas últimas se redujeron $30 \%$ entre 1964 y 1969. En 1972, el año del segundo acuerdo comercial con los soviéticos, el valor de las exportaciones de azúcar a la URSS era inferior al de 1964 (el año del primer acuerdo), mientras que las importaciones de la URSS casi habían duplicado. Sin embargo, con el nuevo acuerdo de 1972 la URSS hizo otras concesiones, estableciendo un precio para el azúcar cubano en línea con la tendencia creciente en el mercado mundial 30 y aceptando que las entregas de Cuba se quedaran bajas para que pudiera vender el azúcar en el mercado mundial aprovechando el incremento de precios (véase cuadro 1 ).

Según Andrew Zimbalist y Susan Eckstein (1987, p. 10), la estrategia basada en el azúcar fracasó porque el precio del producto en el mercado mundial bajó (entre 1966-1968 fue menos de dos centavos por libra). Sin embargo, en la segunda columna del cuadro 1 reportamos las exportaciones cubanas de azúcar en el mercado mundial y en la cuarta la diferencia entre las exportaciones a la URSS según el acuerdo de 1964 y las que efectivamente se realizaron. Las menores entregas a la URSS son de 11400000 toneladas en cinco años, mientras que las ventas en el mercado mundial fueron de 9300000 toneladas. Esto significa que la observación de los dos autores que atribuyen el fracaso de la estrategia de desarrollo entre 1964-1970 a la reducción del precio del azúcar en el mercado mundial no es correcta, ya que Cuba pudo disponer de azúcar para vender en el mercado mundial sólo gracias a la generosidad soviética. Si la URSS hubiese exigido el respeto del acuerdo de 1964, Cuba hubiese tenido que gastar 656000000 de dólares $\$ 1$ en el mercado mundial para cumplir con las entregas pactadas a ese país. ${ }^{32}$

\footnotetext{
${ }^{29}$ En el intento de presionar al gobierno cubano a establecer un sistema de dirección centrado en el cálculo económico utilizado en el resto del campo socialista, en 1967 los soviéticos redujeron las entregas de petróleo a la isla de 20 \% sobre la cantidad pactada (Mesa-Lago y Gil, 1988, p. 65). Las relaciones políticas se volvieron a normalizar el año siguiente, cuando Castro aprobó la invasión soviética de Checoslovaquia y con ellas las entregas soviéticas se restablecieron. Karol (1970) describió la originalidad del intento cubano de construir el socialismo alejándose del camino trazado por la revolución de octubre (por eso lo consideró una herejía), pero advirtió el peligro que representaba la concentración del poder en Castro y su conducta voluntarista en la dirección de la economía, que determinaba decisiones irracionales e inadecuadas.

${ }^{30}$ En el mercado mundial el precio del azúcar pasó de 7.27 centavos por libra en 1972 a 29.66 centavos en 1974 . El precio pagado por la URSS pasó de 6.11 centavos por libra a 19.64 en el mismo periodo (Rodríguez, 1992, p. 65).

${ }^{31}$ Multipliqué las entregas no efectuadas a la URSS por el precio en el mercado mundial, sumando los valores, en el periodo 1964-1970.

${ }^{32}$ Cabe precisar que las exportaciones soviéticas a Cuba siguieron aumentando, de 428000000 de pesos en 1965 a 691000000 en 1970.
} 


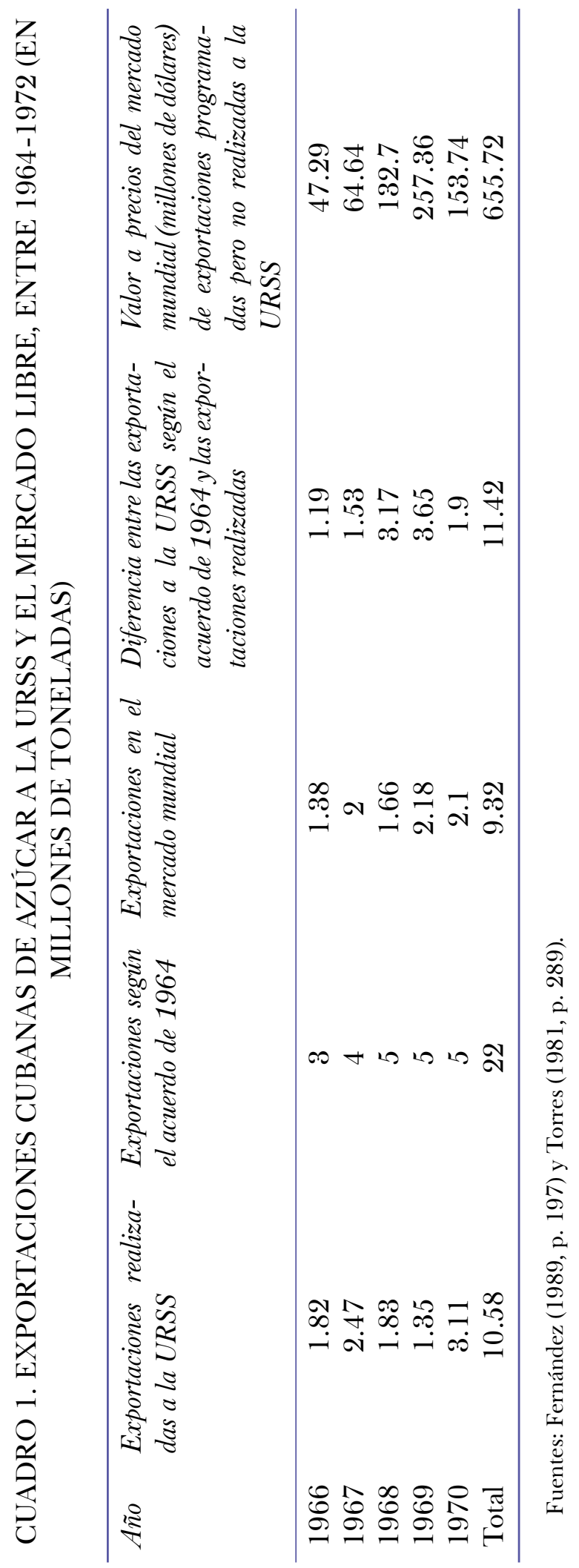


La nueva estrategia de desarrollo decidida en 1964 fue en buena medida financiada por la URSS y fracasó por los errores de política económica cometidos después de 1966 y por rechazar los dos sistemas de dirección existentes, sin sustituirlos por otro ${ }^{33}$ La generosidad y comprensión soviética fueron enormes: la URSS puso a disposición de Cuba los recursos para el desarrollo e intervino para aliviar las consecuencias de los fracasos causados por una conducción irracional y voluntarista de la economía. La ayuda soviética en este periodo hizo posible llevar a cabo importantes inversiones, a pesar de eso la economía se hundió en una grave crisis (véase cuadro 2).

\section{Conclusiones}

Hasta la primera mitad del siglo xx, Cuba era uno de los países más desarrollados de América Latina, pero al mismo tiempo su economía sufría graves desequilibrios. La revolución cubana ha sido un intento radical de liberación definitiva del colonialismo y también de construir un nuevo orden económico y social en beneficio de amplios sectores de la población tradicionalmente discriminados y excluidos.

El afán revolucionario de liberación de las masas se concretó en un esfuerzo redistributivo inicial que generó una base de apoyo consistente al gobierno revolucionario e inevitablemente determinó una ruptura con el gobierno estadunidense y un progresivo acercamiento a la Unión Soviética, hasta la declaración del carácter socialista de la revolución.

Sin embargo, a una primera fase de aplicación de políticas redistributivas tan necesarias en una situación de profundas injusticias y discriminación que afectaba a amplios sectores de la población, no siguió una reorganización del sistema productivo que creara bases firmes para darle cimiento al proceso revolucionario. En los primeros años el gobierno revolucionario trazó una estrategia de desarrollo que pretendía llevar a cabo una profunda transformación económica en poco tiempo, eliminando las dos deformaciones estructurales que Cuba sufría desde que se formó como país independiente en 1902: la dependencia del azúcar y de un solo mercado de destino de este producto. La estrategia fracasó y en 1964 se volvió a centrar en la producción de azúcar, esta vez orientando las exportaciones hacia el mercado soviético, donde se garantizaba a Cuba un precio mayor que en el mercado mundial.

Se trataba de una estrategia mucho más realista, que no fracasó en cuanto tal, pero que resultó impracticable dentro del nuevo modelo económico cubano. A la eliminación de los mecanismos de mercado no correspondió la adopción de la planificación a pesar de la declaración del carácter socialista de la revolución. Eso significó una dirección voluntarista de la economía, donde las decisiones de Castro y las consignas revolucionaria debían por sí sustituir los estímulos a la actividad productiva que el mercado o la planificación centralizada aseguraban. Después de un fecundo debate entre Ernesto Guevara y los socialistas ortodoxos que provenían del Partido Socialista Popular, donde el líder máximo no participó, se prosiguió con un sinnúmero de decisiones económicas irracionales, inspiradas por el mismo Castro, que se alejaban de ambos modelos. Hemos demostrado que, en el análisis de la segunda mitad de la década de 1960, la gran mayoría de los cubanólogos confundió el modelo fidelista aplicado en este periodo con el sistema de financiamiento presupuestario propuesto por el Che Guevara, llegando a calificar como etapa mao-guevarista este periodo. No se trata de peccata minuta, sino de un error en parte propiciado por el mismo Cas-

\footnotetext{
${ }^{33} \mathrm{El}$ mismo Castro (1990, p. 102), comentando las decisiones de política económica tomadas en este periodo, reconoció que "no hemos reconocido el hecho que existen leyes económicas objetivas que tenemos que respetar".
} 
CUADRO 2. VARIACIÓN ESTIMADA DEL PIB PER CÁPITA (1966-1970)

\begin{tabular}{lc}
\hline Años & PIB percápita (variación porcentual) \\
\hline 1966 & -4.1 \\
1967 & 0.2 \\
1968 & 4.4 \\
1969 & -4.3 \\
1970 & -1.9 \\
\hline
\end{tabular}

Fuente: Brundenius (1984, p. 40).

tro, para con ello justificar sus reformas cuyo objetivo final era la total recentralización del poder político en él. Lo que repitió posteriormente en 1986 con el proceso de rectificación y en el 2003 con la batalla de ideas.

La consecuencia de la etapa fidelista en la década de 1960, fue el caos económico, que Castro reconoció en su autocrítica en el Primer Congreso del Partido Comunista Cubano, tachando de idealista su propia conducta en este periodo. Sólo la generosa ayuda soviética evitó el derrumbe del proceso revolucionario, pero eso abrió el camino hacia la dependencia económica, que transformó a Cuba en un país satélite de la Unión Soviética.

\section{LISTA DE REFERENCIAS}

Acosta, J. (1973). Cuba: de la neocolonia a la construcción del socialismo (II). Economía y Desarrollo, 20, 59-117.

Agrupación Católica Universitaria (1972). Encuesta de trabajadores rurales, 1956-1957. Economía y Desarrollo, 12, 188-ss.

Álvarez, L. (1964). Sobre el método de análisis de los sistemas de financiamiento. Cuba Socialista, $35,64-79$.

Azicri, M. (1988). Cuba: politics, economics, and society. Londres: Pinter Publishers.

Azicri, M. (1990). The cuban rectification: safeguarding the revolution while building the future. En S. Halebsky y J. M. Kirk (eds.), Transformation and struggle (pp. 101-113). Nueva York: Praeger.

Banco Nacional de Cuba (1977). Informe Económico. La Habana: Autor.

Bernardo, R. M. (1971). The theory of moral incentives in Cuba. Alabama: University of Alabama Press.

Boorstein, E. (1968). The economic transformation of Cuba: a first-hand account. Nueva York: Monthly Review Press.

Boti, R. (1961). El plan de desarrollo económico de 1962. Cuba Socialista, 1(4), 19.

Brundenius, C. (1984). Revolutionary Cuba, the challenge of economic growth with equity. Boulder, Colo: Westview Press.

Castro, R. F. (1990). Congresos del PCC (1o., 2o. y 3o.: 1975, 1980, 1986). Informe central. La Habana: Política.

Domínguez, J. I. (1978). Cuba: order and revolution. Cambridge: Belknap Press of Harvard University Press. 
Fernández, M. (1989). Cuba y la economía azucarera mundial. La Habana: Pueblo y Educación.

Gey, P. (1987). The Cuban economy under the system of management and planning: success or failure? En P. Gey, J. Kosta, y W. Kaiser (eds.), Crisis and reform in socialist economies (pp. 71-98). Boulder: Westwiew Press.

Guevara, E. (1962). El cuadro, columna vertebral de la revolución. Cuba socialista, 13, 17-22.

Guevara, E. (1964). El cuadro, columna vertebral de la revolución. Cuba socialista, 34, 13-24.

Gutelman, M. (1969). La política agraria de la revolución cubana: 1959-1968. Torino: Einaudi.

Jones, K. (1988). Economic and commercial issues. En S. Wayne y E. Morales (eds.), Subject to solution: problems in Cuban-U.S. relations (pp. 69-77). Boulder: L. Rienner Publishers.

Karol, K. S. (1970). Guerrillas in power: the course of the Cuban revolution. Nueva York: Hill \& Wang. Lataste, A. (1968). Cuba: ihacia una nueva economía politica del socialismo? Santiago: Universitaria.

Martínez, F. (1978). La ley 3 de la Sierra Maestra y la política agraria del ejército rebelde. Economía y Desarrollo, 49.

Mesa-Lago, C. (1968). The labor sector and socialist distribution in Cuba. Nueva York: Frederick A. Praeger.

Mesa-Lago, C. (1981). The economy of socialist Cuba: a two-decade appraisal. Albuquerque: University of New Mexico Press.

Mesa-Lago, C. (1994). Breve historia económica de la Cuba socialista: políticas, resultados y perspectivas. Madrid: Alianza.

Mesa-Lago, C. (2009). Balance económico-social de 50 años de revolución en Cuba. América Latina Hoy, 52, 41-61.

Mesa-Lago, C. (2015). ¿“Cubanólogos” o cubanistas? Temas. Cultura, ideología, sociedad., 81-82, $158-164$.

Mesa-Lago, C. y Gil, F. (1988). Vingt ans de relations economiques entre l' URSS et Cuba. Le courrier des Pays de l' Est, 335, 52-74.

Nova, A. (2008). El sector agropecuario en Cuba. Nueva Sociedad, 216, 77-89.

O'Connor, J. (1970). The origins of socialism in Cuba. Ithaca: Cornell University Press.

Ritter, A. R. M. (1974). The economic development of revolutionary Cuba; strategy and performance. Nueva York: Praeger.

Roca, S. (1977). Cuban economic policy in the 1970s: the trodden paths. En I. L. Horowitz (ed.), Cuban comunism (3a., pp. 83-118). New Brunswick: Transaction Books.

Rodríguez, J. (1987). Agricultural policy and development in Cuba. World Development, 15(1), 23-39. DOI: $10.1016 / 0305-750 X(87) 90100-8$

Rodríguez, J. L. (1988). Crítica a nuestros críticos. La Habana: Ciencias Sociales.

Rodríguez, J. L. (1990). Estrategia del desarrollo económico en Cuba. La Habana: Ciencias Sociales.

Rodríguez, J. L. (1992). Las relaciones económicas entre Cuba y la antigua URSS: evaluación y perspectivas. Cuadernos del Este, 6, 51-66.

Santamaría, A. (1994). Azúcar y Revolución. El sector azucarero de la economía cubana durante los primeros doce años de la Revolución (1959-1970). Revista de Historia Económica/Journal of Iberian and Latin American Economic History, 12(1), 111-141. DOI: 10.1017/ S0212610900004390

Santamaría, A. (2011). Dos siglos de especialización y dos décadas de incertidumbre. La historia económica de Cuba, 1800-2010. En Institucionalidad y desarrollo económico en América Latina (pp. 135-183). Santiago de Chile: Comisión Económica para América Latina y el Caribe. Recuperado de https://repositorio.cepal.org/bitstream/handle/11362/3939/1/S2011122_es.pdf 
Santamaría, A. (2014). La revolución cubana y la economía, 1959-2012. Los ciclos de política y el ciclo azucarero. Anuario de Estudios Americanos, 71(2), 691-723. DOI: 10.3989/aeamer. 2014.2.11

Tablada, C. (1987). El pensamiento económico de Ernesto Che Guevara. La Habana: Casa de las Américas.

Torres, O. E. (1981). El desarrollo de la economía cubana a partir de 1959. Comercio Exterior, 31(3), 284-295.

Valdés, N. P. (1979). The Cuban Revolution: Economic Organization and Bureaucracy. Latin American Perspectives, 6(1), 13-37. DOI: 10.1177/0094582X7900600102

Vilariño, A. (1980). Surgimiento del sistema de dirección de la economía socialista en Cuba. Cuestiones de la Economía Planificada, 3, 60-94.

White, G. (1987). Cuban planning in the mid-1980s: Centralization, decentralization, and participation. World Development, 15(1), 153-161. DOI: 10.1016/0305-750X(87)90108-2

Zimbalist, A. y Eckstein, S. (1987). Patterns of Cuban development: The first twenty-five years. World Development, 15(1), 5-22. DOI: 10.1016/0305-750X(87)90099-4

Zimbalist, A. y Brundenius, C. (1989). The Cuban economy: measurement and analysis of socialist performance. Baltimore: Johns Hopkins University Press. 